\title{
ESTUDO DA FREQÜÊNCIA DE IRRIGAÇÃO, DENSIDADE DE PLANTAS E NÍVEIS DE NITROGÊNIO NA CULTURA DO INHAME ${ }^{1}$
}

\author{
Ladilson de Souza Macêdo ${ }^{2}$ e Elson Soares dos Santos $^{3}$
}

\begin{abstract}
RESUMO
Estudaram-se os efeitos da irrigação, densidade de plantas e adubação nitrogenada sobre a produtividade, peso médio, comprimento e diâmetro de túberas de inhame (Dioscorea cayennensis L.) num solo Podzólico Vermelho-Amarelo de textura arenosa, no município de João Pessoa, PB, nos anos de 1988/89 e 1989/90. Nos experimentos testaram-se três freqüências de irrigação (correspondentes a 40-60, 60-80 e 80-100\% da água disponível para as plantas) duas densidades populacionais (10.417 e 15.625 plantas.ha $\left.^{-1}\right)$ e quatro níveis de nitrogênio $\left(0,50,100\right.$ e $\left.150 \mathrm{~kg} \cdot \mathrm{ha}^{-1}\right)$ no arranjo de parcelas subsubdivididas; a irrigação foi aplicada do início da fase vegetativa (90 dias após o plantio) ao término da floração (210 dias após o plantio). A densidade populacional teve influência significativa na produtividade do inhame e a densidade de 15.625 plantas.ha $^{-1}$ (espaçamento $0,80 \mathrm{~m}$ x $0,80 \mathrm{~m}$ ) apresentou os melhores resultados. Valores máximos de produtividade e peso médio de túberas de inhame foram obtidos com a dose de aproximadamente $90 \mathrm{~kg} \cdot \mathrm{ha}^{-1}$ de nitrogênio. A freqüência de irrigação correspondente a $70 \%$ da água disponível para as plantas, proporcionou resultado satisfatório de desenvolvimento vegetativo e produtividade.
\end{abstract}

Palavras-chave: Dioscorea cayennensis, água disponível, lâmina de água, espaçamento

\section{STUDY OF IRRIGATION FREQUENCY, PLANT DENSITY AND NITROGEN LEVELS ON THE YAM CROP}

\begin{abstract}
The effects of irrigation frequency, plant density and nitrogen fertilization on the productivity, mean weight, length and diameter of yam (Dioscorea cayennensis L.) tubers were evaluated. The experiment was conducted in João Pessoa, Paraíba State, Brazil, in a Red-Yellow Podzolic soil of sandy texture, during 1988-89 and 1989-90. Three irrigation frequencies corresponding to 40-60, $60-80$ and $80-100 \%$ of available water for plants, two plant densities (10.417 and 15.625 plants.ha ${ }^{-1}$ ) and four nitrogen levels $\left(0,50,100\right.$ and $\left.150 \mathrm{~kg} \cdot \mathrm{ha}^{-1}\right)$ in split-split plot were tested. The irrigation water was applied from the beginning of the vegetative phase ( 90 days after planting) until the end of flowering (210 days after planting). The plant density influenced significatively the yam productivity and the density of 15.625 plants.ha ${ }^{-1}$ presented the best results. Maximum yield and mean weight of yam tubers were obtained with approximately $90 \mathrm{~kg} \cdot \mathrm{ha}^{-1}$ of nitrogen. The water level corresponding to $70 \%$ of available water for plants, gave satisfactory results of vegetative development and productivity.
\end{abstract}

Key words: Dioscorea cayennensis, available water, water level, spacing

\footnotetext{
1 Pesquisa realizada com recursos financeiros da EMBRAPA-CNPHortaliças

2 Eng. Agr., M.Sc., Área de Irrigação e Drenagem, EMBRAPA/Empresa Estadual de Pesquisa Agropecuária da Paraíba (EMEPA-PB), CP 275, CEP 58013-290 João Pessoa, PB

3 Eng. Agr., M.Sc., EMEPA-PB
} 


\section{INTRODUÇÃO}

O inhame (Dioscorea cayennensis L.) também conhecido como cará-da-costa, é uma planta herbácea, trepadeira, da família Dioscoreaceae, que vem sendo cultivada com intensidade nos Estados de Pernambuco e Paraíba, maiores produtores da região Nordeste, por se destacar como lavoura de grande importância socio-econômica e, principalmente, por se tornar produto de exportação para os Estados Unidos e países da Europa, o que vem estimulando o aumento da área plantada com o uso de irrigação, incentivando a comercialização interna na entressafra e possibilitando a obtenção de excelente cotação e alta produtividade nos cultivos bem conduzidos.

Trata-se de uma cultura de relativa facilidade de condução e pouco dispendiosa, por não exigir adubações pesadas e por dispensar a necessidade de renovação periódica de sementes (Silva, 1983; Santos, 1996). Todavia, sua produtividade ainda é baixa tanto nos cultivos de inverno como nos irrigados; neste último, o principal fator responsável é o desconhecimento do manejo de água por parte dos irrigantes, haja vista que a água é o componente que limita, com maior intensidade, o potencial produtivo da cultura, sendo indispensável o controle da umidade do solo para o sucesso da agricultura irrigada.

Prejuízos vultosos à agricultura têm sido atribuídos, sobretudo, à má distribuição da precipitação pluvial. Na solução deste problema, a irrigação utilizada adequadamente em complementação às demais atividades agrícolas, torna-se uma técnica de alta relevância, fato este comprovado em outras regiões do mundo, como EUA e Israel, onde a irrigação vem garantindo, em maior escala, a produção agrícola de várias culturas; entretanto, no Brasil os poucos trabalhos de pesquisa relacionados à irrigação do inhame foram desenvolvidos por Santos \& Macêdo (1993), Pereira (1997) e Metri (1997).

O presente trabalho teve como objetivo estudar os efeitos da frequência da irrigação, densidades de plantas e doses crescentes de adubação nitrogenada sobre a produtividade e componentes de produção da cultura do inhame, em um solo Podzólico Vermelho-Amarelo de textura arenosa, da microrregião de João Pessoa, na mesorregião da Mata Paraibana.

\section{MATERIAL E MÉTODOS}

O experimento foi instalado em área experimental da Empresa Estadual de Pesquisa Agropecuária da Paraíba S.A (EMEPA-PB) em João Pessoa, PB, nos anos agrícolas de 1988/89 e 1989/90. O município de João Pessoa está localizado na mesorregião da Mata Paraibana e sua posição geográfica está definida pelas coordenadas $7^{\circ} 06^{\prime} 57^{\prime \prime} \mathrm{S}, 34^{\circ} 53^{\prime} 14^{\prime \prime} \mathrm{W}$. Gr. e altitude de 30m; a precipitação pluviométrica média anual é de $1.981 \mathrm{~mm}$, umidade relativa do ar média anual de $80 \%$ e temperatura média anual máxima de $30^{\circ} \mathrm{C}$ e mínima de $23^{\circ} \mathrm{C}$ (período de $1988 / 1990$ ). A distribuição da precipitação pluviométrica ocorrida no período de condução dos experimentos e as fases de crescimento da cultura do inhame para subsidiar no manejo da irrigação podem ser visualizados na Figura 1.

O solo utilizado foi um Podzólico Vermelho-Amarelo, de textura arenosa, baixa fertilidade natural e baixa capacidade de retenção de água, conforme resultado das análises física e química: pH em água $(1: 2,5)=4,7$ (acidez elevada); $\mathrm{Al}^{3+}$

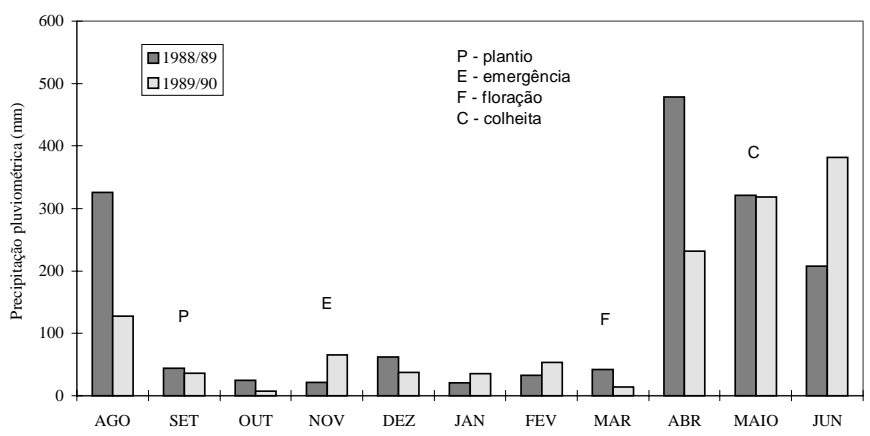

Figura 1. Distribuição da precipitação pluviométrica no período de condução dos experimentos

trocável $=0,4 \mathrm{cmol}_{c} \cdot \mathrm{kg}^{-1}$ de TFSA (médio); $\mathrm{Ca}^{2+}+\mathrm{Mg}^{2+}$ trocável $=1,4 \mathrm{cmol} \cdot \mathrm{kg}^{-1} \mathrm{de}$ TFSA (baixo) $\mathrm{P}$ disponível $=2,0 \mathrm{mg} \cdot \mathrm{dm}^{-3}$ (baixo); $\mathrm{K}^{+}$disponível = 10,0 mg.dm ${ }^{-3}$ (baixo); matéria orgânica $=0,9 \%$ (baixa); capacidade de campo $=7,2 \%$; ponto de murcha permanente $=3,3 \%$ e densidade aparente $=1,6 \mathrm{~kg} \cdot \mathrm{dm}^{-3}$. As análises foram realizadas no Laboratório de Fertilidade do Solo, do Centro Nacional de Pesquisa de Algodão (CNPA). A interpretação da análise química teve como base os níveis de fertilidade do solo publicados por Lopes (1989).

O solo foi preparado mecanicamente com gradagens de disco cruzadas. A adubação no plantio constou da aplicação por cova, de esterco de bovino na dose de $1,5 \mathrm{~kg}$, no espaçamento de menor densidade (10.417 plantas.ha ${ }^{-1}$ ) e de 1,0kg no espaçamento de maior densidade ( 15.625 plantas.ha $^{-1}$ ). Realizou-se uma adubação básica com $120 \mathrm{~kg} \cdot h a^{-1}$ de $\mathrm{P}_{2} \mathrm{O}_{5}$ e $60 \mathrm{~kg} \cdot \mathrm{ha}^{-1}$ de $\mathrm{K}_{2} \mathrm{O}$ nas formas de superfosfato triplo e cloreto de potássio, respectivamente, para corrigir a deficiência desses nutrientes no solo. A adubação nitrogenada foi parcelada em $20 \%$ no início da brotação, $40 \%$ com o aparecimento das primeiras folhas e $40 \%$ no aparecimento dos ramos primários, estágios de crescimento que ocorrem aos 30, 60 e 90 dias após o plantio, respectivamente.

O plantio foi realizado no mês de setembro de cada ano agrícola e a colheita no mês de abril do ano seguinte. Antes do plantio, o solo foi devidamente umedecido com uma lâmina de água suficiente para elevar os primeiros $50 \mathrm{~cm}$ à capacidade de campo, sendo realizadas irrigações leves até os 90 dias, com uma lâmina de $12 \mathrm{~mm}$ a intervalos de cinco dias, para proporcionar condições adequadas de temperatura e umidade à germinação das túberas-semente. No primeiro ano, foi utilizado o sistema de plantio em cova alta (matumbos) com dimensão de $40 \mathrm{~cm}$ x $40 \mathrm{~cm}$ x $40 \mathrm{~cm}$ e sementes "lisas"; no segundo ano, foi utilizado o sistema de plantio em camalhão (leirão) e sementes "capadas"; as sementes foram da cultivar Da Costa, previamente selecionadas e plantadas, com peso de 250 a $300 \mathrm{~g}$.

O delineamento experimental usado foi o de blocos ao acaso, com arranjo de parcelas subsubdivididas, com quatro repetições. Cada parcela principal correspondeu a uma frequiência de irrigação (correspondente a 40-60, 60-80 e 80-100\% da água disponível para as plantas), constituída de duas subparcelas, correspondentes às densidades populacionais 10.417 plantas.ha $^{-1}(1,20 \mathrm{~m} \mathrm{x} 0,80 \mathrm{~m})$ e 15.625 plantas.ha $^{-1}(0,80 \mathrm{~m} \times 0,80 \mathrm{~m})$; cada subparcela foi formada por quatro subsubparcelas, correspondentes aos níveis de nitrogênio $\left(0,50,100\right.$ e $\left.150 \mathrm{~kg} \cdot \mathrm{ha}^{-1}\right)$. A unidade experimental constou de quatro fileiras de plantas, ocupando uma área de $30,72 \mathrm{~m}^{2}(6,40 \mathrm{~m} \times 4,80 \mathrm{~m})$ considerada útil; as parcelas principais 
do experimento foram distanciadas em $15 \mathrm{~m}$ para evitar interferência de água entre os tratamentos de irrigação. Utilizou-se o método de irrigação por aspersão, com dois ramais e distribuição da precipitação na forma quadrática $12 \mathrm{~m}$ x $12 \mathrm{~m}$.

O complexo florístico daninho foi controlado mediante cinco capinas manuais com enxada. Não foi verificada a ocorrência de pragas nem doenças, a nível significativo, dispensando-se o controle fitossanitário com produtos químicos.

As variáveis estudadas foram: produtividade $\left(\mathrm{t}^{\mathrm{h}} \mathrm{h}^{-1}\right)$, peso médio $(\mathrm{kg})$, comprimento $(\mathrm{cm})$ e diâmetro $(\mathrm{mm})$ das túberas produzidas. O comprimento constou do valor médio de três túberas amostradas casualmente em cada parcela principal, enquanto o diâmetro consistiu da média diametral das partes superior, meio e inferior dessa mesma amostragem de túberas; os dados foram avaliados estatisticamente por meio da análise de variância, de acordo com Federer (1980) usando-se o programa NTIA da EMBRAPA, descrito por Paniago et al. (1995); as médias foram comparadas pelo teste de Duncan, a nível de $5 \%$ de probabilidade.

\section{RESULTADOS E DISCUSSÃO}

Os quadrados médios das análises de variância, as médias e os coeficientes de variação para as variáveis em estudo, que serviram de base para avaliação do primeiro ano do experimento (1988/89) estão apresentados na Tabela 1, cujos resultados revelam que as freqüências de irrigação influenciaram significativamente $(\mathrm{P}<0,05)$ a produtividade, o comprimento $\mathrm{e}$ o diâmetro das túberas de inhame, não exercendo influência sobre o seu peso médio. O efeito da densidade de plantas foi significativo $(\mathrm{P}<0,01)$ unicamente para a produtividade de inhame. A inexistência de diferenças significativas entre densidades de plantas sobre o comprimento e o diâmetro das túberas está relacionada, provavelmente, ao tamanho da amostra usado para determinação dessas variáveis, as quais foram mensuradas em apenas três túberas tomadas aleatoriamente em cada parcela principal.

Tabela 1. Análises de variância da produtividade $\left(\right.$ t.ha $\left.^{-1}\right)$, peso médio $(\mathrm{kg})$, comprimento $(\mathrm{cm})$ e diâmetro $(\mathrm{mm})$ de túberas de inhame produzidas sob regime de irrigação.

\begin{tabular}{|c|c|c|c|c|c|c|c|}
\hline \multirow[t]{2}{*}{ Fontes de variação } & \multirow[t]{2}{*}{ GL } & \multicolumn{6}{|c|}{ Quadrados médios } \\
\hline & & Produtividade & Peso $n$ & nédio & Comprim & nento & Diâmetro \\
\hline Blocos & 3 & $6,268 \mathrm{~ns}$ & 0,303 & $\mathrm{~ns}$ & 55,622 & $* *$ & $219,788 *$ \\
\hline Frequiência de irrigação $=F$ & 2 & $296,526 *$ & 0,561 & $\mathrm{~ns}$ & 28,135 & $*$ & $288,448 *$ \\
\hline Resíduo (a) & 6 & 31,059 & 0,239 & & 4,413 & & 39,851 \\
\hline Densidade de plantas $=\mathrm{D}$ & 1 & $513,399 * *$ & 0,629 & $\mathrm{~ns}$ & 12,760 & $\mathrm{~ns}$ & $75,260 \mathrm{n}$ \\
\hline DxF & 2 & $16,190 \mathrm{~ns}$ & 0,539 & $\mathrm{~ns}$ & 29,385 & $*$ & $48,573 \mathrm{n}$ \\
\hline Resíduo (b) & 9 & 16,879 & 0,161 & & 4,538 & & 114,191 \\
\hline Níveis de nitrogênio $=\mathrm{N}$ & 3 & $26,475 \mathrm{~ns}$ & 0,096 & ns & 57,844 & * & $482,344 * *$ \\
\hline Linear & 1 & $31,877 \mathrm{~ns}$ & 0,177 & $\mathrm{~ns}$ & 162,169 & ** & $548,269 * *$ \\
\hline Quadrático & 1 & $4,808 \mathrm{~ns}$ & 0,001 & $\mathrm{~ns}$ & 4,594 & $\mathrm{~ns}$ & 846,094 ** \\
\hline Cúbico & 1 & $42,739 \mathrm{~ns}$ & 0,109 & ns & 6,769 & $\mathrm{~ns}$ & $52,669 \mathrm{n}$ \\
\hline $\mathrm{N} \times \mathrm{F}$ & 6 & $36,736 *$ & 0,438 & * & 9,677 & $\mathrm{~ns}$ & $27,365 \mathrm{n}$ \\
\hline $\mathrm{N} \times \mathrm{D}$ & 3 & $1,614 \mathrm{~ns}$ & 0,214 & ns & 9,955 & $\mathrm{~ns}$ & $23,677 \mathrm{n}$ \\
\hline $\mathrm{N} \times \mathrm{D} \times \mathrm{F}$ & 6 & $5,341 \mathrm{~ns}$ & 0,194 & $\mathrm{~ns}$ & 18,538 & $\mathrm{~ns}$ & $83,073 \mathrm{n}$ \\
\hline Resíduo (c) & 54 & 15,855 & 0,185 & & 19,168 & & 61,733 \\
\hline $\mathrm{CV}(\mathrm{a}) \%$ & & 34,47 & 24,57 & & 7,65 & & 6,67 \\
\hline $\mathrm{CV}(\mathrm{b}) \%$ & & 25,41 & 20,16 & & 7,76 & & 11,30 \\
\hline $\mathrm{CV}(\mathrm{c}) \%$ & & 24,62 & 21,61 & & 15,95 & & 8,31 \\
\hline
\end{tabular}

e* Significativo a 5 e $1 \%$ de probabilidade, respectivamente (teste $\mathrm{F}$ )

(ns) Não significativo

As análises de variância para produtividade e peso médio das túberas de inhame não indicaram significância estatística $(\mathrm{P}>0,05)$ para os efeitos das doses de nitrogênio, quando analisadas isoladamente; este resultado pode estar associado ao fato da adubação nitrogenada ter sido efetuada próximo à época da irrigação subseqüente, provocando a lixiviação do elemento e tornando sem efeito sua absorção pelas plantas. Com relação às dimensões das túberas, verificou-se efeito linear significativo das doses de nitrogênio para comprimento, e quadrático para diâmetro, a nível de 5\% de probabilidade.

A interação entre doses de nitrogênio e frequiência de irrigação foi significativa $(\mathrm{P}<0,05)$ sobre a produtividade e o peso médio das túberas. Para se verificar as causas desta interação, fez-se um estudo de regressão das doses de nitrogênio em cada lâmina de irrigação, verificando-se que na lâmina de 40-60\% da água disponível (AD) para as plantas ocorreu diminuição acentuada na produtividade e no peso médio, em função dos níveis crescentes de nitrogênio aplicados ao solo, expressa pelas seguintes equações: $Y_{1}=15,686-0,03758 X$ $\left(R^{2}=89 \%\right)$ e $Y_{2}=2,108-0,00354 X\left(R^{2}=92 \%\right)$ em que $Y_{1}$ e $Y_{2}$ são a produtividade $\left(\mathrm{t}^{\mathrm{h}} \mathrm{a}^{-1}\right)$ e o peso médio das túberas $(\mathrm{kg})$ respectivamente, e $\mathrm{X}$ é a quantidade aplicada de nitrogênio $\left(\mathrm{kg} \mathrm{ha}^{-1}\right)$; na frequiência correspondente a de 60-80 \% da $\mathrm{AD}$ também ocorreu decréscimo na produtividade e no peso médio das túberas, com o aumento dos níveis de nitrogênio, porém menos acentuado, expresso pelas seguintes equações: $\mathrm{Y}_{1}=18,067-0,01746 \mathrm{X}\left(\mathrm{R}^{2}=53 \%\right)$ e $\mathrm{Y}_{2}=2,157-0,00206 \mathrm{X}$ $\left(\mathrm{R}^{2}=97 \%\right)$, respectivamente; na lâmina correspondente a 80$100 \%$ da $\mathrm{AD}$ aconteceu o contrário, em que a produtividade e o peso médio das túberas aumentaram lentamente com as doses crescentes de nitrogênio, cujos incrementos foram expressos pelas equações: $Y_{1}=17,039+0,02458 X\left(R^{2}=75 \%\right)$ e $Y_{2}=1,866+0,00322 X\left(R^{2}=94 \%\right)$ respectivamente.

Não foi detectada significância estatística $(P>0,05)$ das interações doses de nitrogênio $\mathrm{x}$ densidade de plantas e doses de nitrogênio $x$ densidade de plantas $x$ lâminas de irrigação sobre as variáveis avaliadas; do mesmo modo, a interação densidade de plantas $\mathrm{x}$ lâmina de irrigação não foi significativa $(\mathrm{P}>0,05)$ sobre a produtividade, peso médio e diâmetro das túberas, sendo-o apenas sobre o comprimento do inhame.

A análise da Tabela 2 permite verificar, no primeiro ano do experimento, que a frequiência de irrigação de $80-100 \%$ da água disponível para as plantas promoveu produtividade média mais elevada não diferindo, porém, estatisticamente $(\mathrm{P}>0,05)$ da produtividade obtida com a lâmina de $60-80 \%$ da $\mathrm{AD}$; resposta idêntica foi observada para o comprimento e diâmetro das túberas. Com relação a peso médio das túberas, não houve diferenças significativas $(P>0,05)$ entre frequiências de irrigação e esses resultados permitem indicar, para a cultura do inhame, uma irrigação equivalente, em média, a 70\% da AD para as plantas, com frequiência em média de três dias; este resultado está de acordo com Santos \& Macêdo (1993) e Santos (1996).

Tabela 2. Resultados médios de produtividade, peso, comprimento e diâmetro de túberas de inhame, em função da freqüência de irrigação e da densidade de plantas

\begin{tabular}{lcccc}
\hline Tratamentos & $\begin{array}{c}\text { Produtividade } \\
\left(\text { t.ha }^{-1}\right)\end{array}$ & $\begin{array}{c}\text { Peso médio } \\
(\mathrm{kg})\end{array}$ & $\begin{array}{c}\text { Comprimento } \\
(\mathrm{cm})\end{array}$ & $\begin{array}{c}\text { Diâmetro } \\
(\mathrm{mm})\end{array}$ \\
\hline Freqüënc1a de Irrigaçáo (\% AD) & $12,88 \mathrm{~b}$ & $1,84 \mathrm{a}$ & $26,63 \mathrm{~b}$ & $91,1 \mathrm{~b}$ \\
$\quad 40-60$ & $16,76 \mathrm{ab}$ & $2,01 \mathrm{a}$ & $27,25 \mathrm{ab}$ & $96,7 \mathrm{a}$ \\
$60-80$ & $18,88 \mathrm{a}$ & $2,10 \mathrm{a}$ & $28,47 \mathrm{a}$ & $95,8 \mathrm{ab}$ \\
$80-100$ & & & & \\
Densidade populacional (plantas.ha $\left.{ }^{-1}\right)$ & $13,86 \mathrm{~b}$ & $2,07 \mathrm{a}$ & $27,81 \mathrm{a}$ & $93,7 \mathrm{a}$ \\
$\quad 10.417(1,20 \mathrm{~m} \times 0,80 \mathrm{~m})$ & $18,49 \mathrm{a}$ & $1,90 \mathrm{a}$ & $27,08 \mathrm{a}$ & $95,4 \mathrm{a}$ \\
$15.625(0,80 \mathrm{~m} \times 0,80 \mathrm{~m})$ & 16,17 & 1,99 & 27,45 & 94,6 \\
Média geral & & &
\end{tabular}

Médias com a mesma letra, nas colunas e no mesmo tratamento, não diferem significativamente, pelo teste de Duncan a $5 \%$

$\% \mathrm{AD}=\%$ Água disponível para as plantas 
A densidade populacional de 15.625 plantas.ha $^{-1}$ proporcionou a mais elevada produtividade $\left(18,49 \mathrm{t}^{\left.\mathrm{h} \mathrm{h}^{-1}\right)} \mathrm{em}\right.$ relação à densidade de 10.417 plantas.ha ${ }^{-1}\left(13,86\right.$ t.ha $\left.^{-1}\right)$, com uma diferença significativa $(\mathrm{P}<0,05)$ de 4,63t.ha ${ }^{-1}$. Este comportamento é função do maior número de plantas proporcionado pelo adensamento entre fileiras de $1,20 \mathrm{~m}$ para $0,80 \mathrm{~m}$. Com relação às demais variáveis, não se constatou diferenças significativas $(\mathrm{P}>0,05)$ entre densidades de plantas. Segundo Janick (1968) o aumento da densidade populacional pode atingir um ponto no qual as plantas começam a competir por luz, água, $\mathrm{CO}_{2}$ e nutrientes, resultando em decréscimo da produção por planta. As reações são expressas pelas partes da planta, através da diminuição dos tubérculos, das folhas, flores e outros, que reagem, de modo diferente, as pressões de uma população crescente. No presente trabalho esse ponto não foi atingido e, portanto, o aumento da densidade populacional não exerceu efeito negativo no comportamento produtivo das plantas, visto que ocorreu maior produtividade na maior densidade de plantas.

Pode-se observar, na Figura 2, que as produtividades médias aumentaram de acordo com o incremento das doses de nitrogênio, atingindo um ponto de máxima produtividade (26,94 t.ha $\left.{ }^{-1}\right)$ estimado com a dose de $86 \mathrm{~kg} \cdot \mathrm{ha}^{-1}$ de nitrogênio, em seguida decaindo.

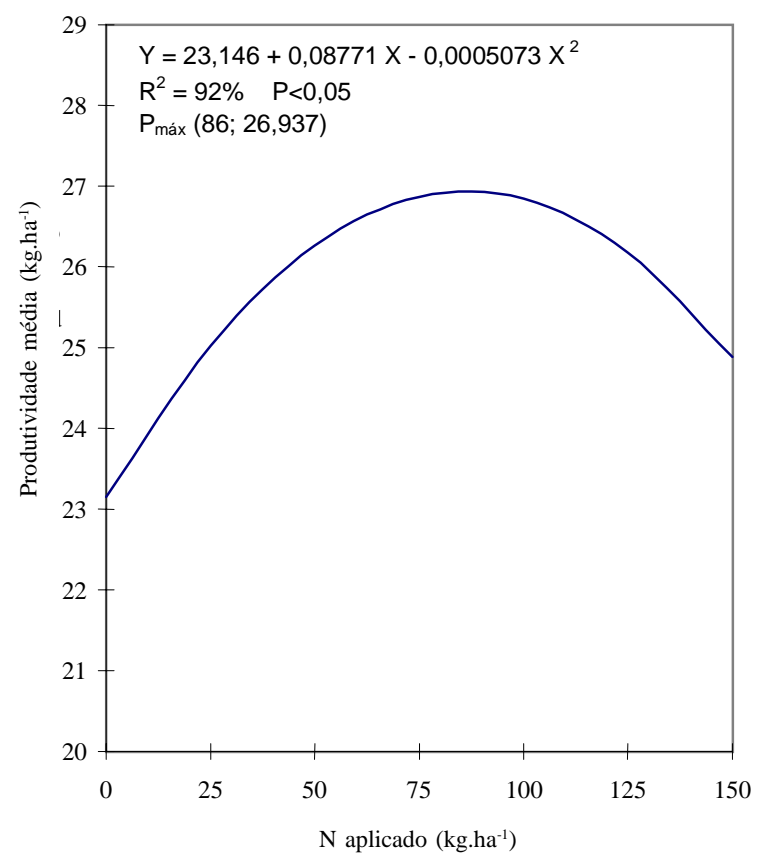

Figura 2. Relação entre doses de nitrogênio e produtividade média de inhame irrigado no ano de 1989/90

No segundo ano (1989/90) os resultados das análises de variância da produtividade, peso médio, comprimento e diâmetro das túberas de inhame produzidas, sob regime de irrigação, são apresentados na Tabela 3, cujos dados revelam que as freqüências de irrigação influenciaram significativamente, a nível de $5 \%$, unicamente a produtividade. A densidade de plantas afetou significativamente, a nível de $1 \%$ de probabilidade, a produtividade e, a nível de $5 \%$, o peso médio das túberas, não afetando, no entanto, o comprimento nem o diâmetro das mesmas.

Tabela 3. Análises de variância da produtividade (t.ha $\left.{ }^{-1}\right)$, peso médio $(\mathrm{kg})$, comprimento $(\mathrm{cm})$ e diâmetro $(\mathrm{mm})$ de túberas de inhame produzidas sob regime de irrigação.

\begin{tabular}{lccccc}
\hline Fontes de variação & GL & \multicolumn{5}{c}{ Quadrados médios } \\
\cline { 3 - 6 } & & Produtividade & Peso médio & Comprimento & Diâmetro \\
\hline Blocos & 3 & $247,430 \mathrm{~ns}$ & $1,891 \mathrm{~ns}$ & $77,083 *$ & $378,288 \mathrm{~ns}$ \\
Freqüência de irrigação = F & 2 & $311,057 *$ & $1,399 \mathrm{~ns}$ & $15,385 \mathrm{~ns}$ & $201,125 \mathrm{~ns}$ \\
Resíduo (a) & 6 & 55,750 & 0,440 & 8,135 & 110,069 \\
Densidade de plantas = D & 1 & $1.212,297 * *$ & $1,015 *$ & $3,375 \mathrm{~ns}$ & $133,010 \mathrm{~ns}$ \\
D x F & 2 & $8,601 \mathrm{~ns}$ & $0,025 \mathrm{~ns}$ & $9,594 \mathrm{~ns}$ & $16,792 \mathrm{~ns}$ \\
Resíduo (b) & 9 & 11,871 & 0,159 & 24,660 & 56,114 \\
Níveis de nitrogênio = N & 3 & $70,550 \mathrm{~ns}$ & $0,644 \mathrm{~ns}$ & $33,806 \mathrm{~ns}$ & $318,760 *$ \\
$\quad$ Linear & 1 & $40,439 \mathrm{~ns}$ & $0,539 \mathrm{~ns}$ & $81,675 \mathrm{~ns}$ & $674,502 * *$ \\
$\quad$ Quadrático & 1 & $154,394 *$ & $1,167 *$ & $10,667 \mathrm{~ns}$ & $250,260 *$ \\
$\quad$ Cúbico & 1 & $16,816 \mathrm{~ns}$ & $0,225 \mathrm{~ns}$ & $9,075 \mathrm{~ns}$ & $31,519 \mathrm{~ns}$ \\
N x L & 6 & $19,533 \mathrm{~ns}$ & $0,173 \mathrm{~ns}$ & $50,899 \mathrm{~ns}$ & $30,208 \mathrm{~ns}$ \\
N x D & 3 & $1,169 \mathrm{~ns}$ & $0,037 \mathrm{~ns}$ & $12,736 \mathrm{~ns}$ & $35,233 \mathrm{~ns}$ \\
N x D x F & 6 & $3,579 \mathrm{~ns}$ & $0,018 \mathrm{~ns}$ & $23,080 \mathrm{~ns}$ & $40,847 \mathrm{~ns}$ \\
Resíduo (c) & 54 & 26,321 & 0,285 & 30,907 & 46,527 \\
\hline CV(a) \% & \multicolumn{7}{c}{29,52} & 25,03 & 9,44 & 11,47 \\
CV(b) \% & 13,62 & 15,05 & 16,44 & 8,19 \\
CV(c) \% & & 20,29 & 20,15 & 18,40 & 7,45 \\
\hline
\end{tabular}

* e ** Significativo a 5 e $1 \%$ de probabilidade, respectivamente (teste $\mathrm{F}$ ) (ns) Não significativo

Houve efeito quadrático significativo a nível de 5\% de probabilidade, das doses de nitrogênio sobre a produtividade, o peso médio e o diâmetro das túberas de inhame. Não foi verificado efeito significativo $(\mathrm{P}>0,05)$ das interações densidade de plantio $\mathrm{x}$ frequiências de irrigação, doses de nitrogênio $\mathrm{x}$ freqüências de irrigação, doses de nitrogênio $x$ densidade de plantas, doses de nitrogênio $\mathrm{x}$ densidade de plantas $\mathrm{x}$ freqüências de irrigação, indicando que esses fatores agiram independentemente um do outro.

$\mathrm{Na}$ Tabela 4, verifica-se que, no segundo ano do experimento, a freqüência de irrigação correspondente a 60-80\% da AD proporcionou a mais elevada produtividade $\left(27,77\right.$ t.ha $\left.{ }^{-1}\right)$ sem, contudo, diferir significativamente $(\mathrm{P}>0,05)$ da freqüência de 80-100\% da AD com 26,31 t.ha ${ }^{-1}$, porém superando estatisticamente $(\mathrm{P}<0,05)$ a produtividade obtida na freqüência de irrigação de 40-60\% da $\mathrm{AD}\left(21,79\right.$ t.ha $\left.^{-1}\right)$; esses resultados permitem indicar, para a cultura do inhame, em termos de produtividade máxima econômica, uma irrigação equivalente, em média, a $70 \%$ da água disponível para as plantas.

Tabela 4. Resultados médios ${ }^{1}$ de produtividade, peso, comprimento e diâmetro de túberas de inhame, em função das freqüências de irrigação e da densidade de plantas.

\begin{tabular}{lcccc}
\hline Tratamentos & $\begin{array}{c}\text { Produtividade } \\
\left(\mathrm{t} . \mathrm{ha}^{-1}\right)\end{array}$ & $\begin{array}{c}\text { Peso médio } \\
(\mathrm{kg})\end{array}$ & $\begin{array}{c}\text { Comprimento } \\
(\mathrm{cm})\end{array}$ & $\begin{array}{c}\text { Diâmetro } \\
(\mathrm{cm})\end{array}$ \\
\hline Freqüência de irrigação $\left(\% \mathrm{AD}^{2}\right)$ & & & & \\
$\quad 40-60$ & $21,79 \mathrm{~b}$ & $2,41 \mathrm{a}$ & $30,78 \mathrm{a}$ & $90,6 \mathrm{a}$ \\
$\quad 20-80$ & $27,77 \mathrm{a}$ & $2,80 \mathrm{a}$ & $30,41 \mathrm{a}$ & $94,3 \mathrm{a}$ \\
$\quad 80-100$ & $26,31 \mathrm{ab}$ & $2,73 \mathrm{a}$ & $29,44 \mathrm{a}$ & $89,5 \mathrm{a}$ \\
Densidade populacional (plantas.ha $\left.{ }^{-1}\right)$ & & & & \\
$\quad 21,74 \mathrm{~b}$ & $2,75 \mathrm{a}$ & $30,02 \mathrm{a}$ & $92,7 \mathrm{a}$ \\
$\quad 10.417(1,20 \mathrm{~m} \times 0,80 \mathrm{~m})$ & $28,84 \mathrm{a}$ & $2,54 \mathrm{~b}$ & $30,40 \mathrm{a}$ & $90,3 \mathrm{a}$ \\
$\quad 15.625(0,80 \mathrm{~m} \times 0,80 \mathrm{~m})$ & 25,29 & 2,65 & 30,21 & 91,5 \\
\hline
\end{tabular}

${ }^{1}$ Médias com a mesma letra, nas colunas e no mesmo tratamento, não diferem significativamente, pelo teste de Duncan a 5\%

$2 \% \mathrm{AD}=$ Água disponível para as plantas

Constata-se ainda, nesta mesma Tabela, que a melhor produtividade $\left(28,84\right.$ t.ha $\left.^{-1}\right)$ foi obtida na densidade de 15.625 plantas.ha ${ }^{-1}$, superando significativamente $(\mathrm{P}<0,05)$ em 7,10 t.ha $^{-1}$ aquela obtida na densidade de 10.417 plantas.ha ${ }^{-1}\left(21,74\right.$ t.ha $\left.^{-1}\right)$. Esta superioridade pode ser atribuída à maior população de plantas proporcionada pelo adensamento entre fileiras no espaçamento de $0,40 \mathrm{~m}$, e o resultado indica que a produtividade 
das plantas aumentou na proporção direta do aumento populacional, concordando com a observação feita por Janick (1968).

O peso médio das túberas foi maior na densidade de 10.417 plantas.ha $\mathrm{ha}^{-1}(2,75 \mathrm{~kg})$ em relação à densidade de 15.625 plantas.ha-1 $(2,54 \mathrm{~kg})$ com uma diferença significativa $(\mathrm{P}<0,05)$ de $0,21 \mathrm{~kg}$. Este resultado está relacionado ao maior adensamento da lavoura, no espaçamento de $0,80 \mathrm{~m}$ x $0,80 \mathrm{~m}$. Segundo Janick (1968) quando se aumenta a densidade populacional atinge-se um ponto no qual as plantas começam a competir por alguns fatores essenciais de crescimento, resultando em decréscimo da produtividade por planta. Semelhante ao Experimento 1988/89 não houve diferenças significativas $(\mathrm{P}>0,05)$ entre densidade de plantas sobre o comprimento nem o diâmetro das túberas, cujas médias foram de $30,21 \mathrm{~cm}$ e $9,15 \mathrm{~cm}$, respectivamente, estando as causas deste resultado associadas, provavelmente, ao tamanho da amostra adotado para determinação dessas dimensões.

A exemplo do que ocorreu com a produtividade, os pesos médios aumentaram com as doses crescentes de nitrogênio, atingindo um ponto de máximo $(2,27 \mathrm{~kg})$ estimado com a dose de 93 kg.ha-1 de nitrogênio (Figura 3).

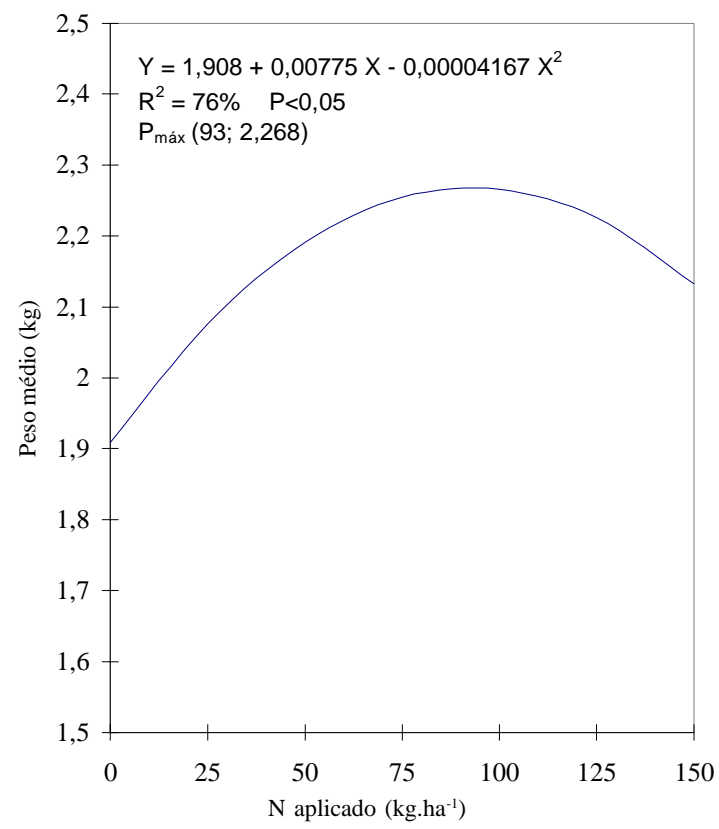

Figura 3. Relação entre doses de nitrogênio e peso médio das túberas de inhame irrigado no ano de 1989/90

\section{CONCLUSÕES}

1. A aplicação da freqüência de irrigação correspondente a $70 \%$ da água disponível para as plantas proporcionou resultados satisfatórios de desenvolvimento vegetativo e produtividade da cultura do inhame, em solo de textura arenosa.

2. A densidade de plantio de 15.625 plantas.ha $^{-1}$ (espaçamento de $0,80 \mathrm{~m} \times 0,80 \mathrm{~m}$ ) proporcionou maior produtividade no cultivo do inhame irrigado.

3. O aumento das doses de nitrogênio provocou incremento quadrático significativo nos valores de produtividade e peso médio de túberas de inhame, atingindo máximos na dose de aproximadamente $90 \mathrm{~kg} \cdot \mathrm{ha}^{-1}$ de nitrogênio.

4. Nos plantios irrigados sugere-se a aplicação de água de irrigação a partir do surgimento das primeiras folhas da planta até o término da floração.

\section{REFERÊNCIAS BIBLIOGRÁFICAS}

FEDERER, W.T. Statistical design: treatment design and derived experiment designs. Brasília: EMBRAPA, 1980. 236p.

JANICK, J. A ciência da horticultura. São Paulo: Freitas Bastos, 1968. p.277-286.

LOPES, A.S. Manual de fertilidade do solo. São Paulo: ANDA/POTAFOS, 1989. 153p. il.

METRI, J.E. da C. Estimativas preliminares das necessidades hídricas do inhame (Dioscorea cayennensis Lam.). Campina Grande: CCT/UFPB, 1997. 113p. Tese de Doutorado.

PANIAGO, C.F.A.; ANDRADE, D.P. de; TSURUTA, J.H.; CAMARGO NETO, J.; MOURA, M.F. Software científicoNTIA: versão 2.0. Campinas: EMBRAPA-NTIA, 1995, 8 disquetes $31 / 2$ ".

PEREIRA, J.R.B. Estudo da umidade do solo na fase de germinação do cará-da-costa (Dioscorea cayennensis Lam.). Coeficientes hídricos. Campina Grande: CPGEAG/ CCT/UFPB, 1997. 89p. Dissertação de Mestrado.

SANTOS, E.S. dos. Inhame (Dioscorea spp); aspectos básicos da cultura. João Pessoa, PB: EMEPA-PB, SEBRAE, 1996. 158p. il.

SANTOS, E.S. dos; MACÊDO, L. de. S. Estudo da cultura do inhame (Dioscorea sp.) na Paraíba. João Pessoa: EMEPA-PB, FBB, 1993. 113p. (Relatório Final de Projeto).

SILVA, A.A. de. Cultura do cará-da-costa: Dioscorea cayennensis Lam. var. Rotundata Poir. 2 ed. Fortaleza: BNB-ETENE, 1983. 73p. il. 